\section{Neglected conditions}

Neuroendocrine tumours: The day after the third annual Worldwide NET (neuroendocrine tumour) Cancer Awareness Day, Kelsall ${ }^{1}$ pointed out the multiplication of disease recognition days in a $C M A J$ editorial and called for "articles on neglected conditions that may benefit from increased attention."

Despite the recent rise in incidence of this rare malignancy, patients with neuroendocrine tumours experience diagnostic delays of up to seven years after presentation of initial symptoms. This delay may be due to rarity, nonspecific symptoms, unknown risk factors or a lack of awareness among primary care and specialized physicians. ${ }^{2,3}$ In Ontario, patients with neuroendocrine tumours visited primary care physicians a median of 14 times before receiving a diagnosis. ${ }^{3,4}$ The combination of slow progression and hormonal production in advanced neuroendocrine tumours produces debilitating symptoms, which lead to substantial deterioration of quality of life.

Neuroendocrine tumours represent a rare "chronic cancer" that forces patients to cope with steady debilitation. Beyond fragmented care, patient support is compromised because little information on prognosis and treatment is available to physicians. Large-scale analyses of epidemiology, behaviour, and health care delivery and utilization surrounding neuroendocrine tumours are paramount to patients and physicians in terms of timely diagnosis and delivery of effective therapies.

Neuroendocrine tumours must cease to be a diagnosis of exclusion that is established after years of investigation. We strongly believe that research is desperately needed to create targeted programs to raise awareness in the medical community and to improve outcomes and physician and patient experience. A comprehensive review of the current knowledge surrounding neuroendocrine tumours is the first step. It is often said that when hearing hoofbeats, one should think horses, not zebras ... but sometimes one is not far from the Serengeti and has to know about zebras.

\section{Julie Hallet MD, Simron Singh MD MPH, Calvin H.L. Law MD MPH}

Division of General Surgery (Hallet, Law), University of Toronto; Divisions of Medical Oncology \& Hematology (Singh) and Surgical Oncology (Law), Odette Cancer Centre, Sunnybrook Health Sciences Centre, Toronto, Ont.

\section{References}

1. Kelsall D. With a disease for every day, who will care for the orphans? CMAJ 2013;185:1475.

2. Moldin IM, Champaneria MC, Chan AKC, et al. A three-decade analysis of 3911 small intestinal neuroendocrine tumors: the rapid pace of no progress. Am J Gastroenterol 2007;102:1464-73.

3. Singh S, Saskin R, Lin N, et al. 2012 Neuroendocrine Tumor Symposium abstracts: patterns of care of neuroendocrine cancer in Ontario: health services delivery. Vancouver (WA): North American Neuroendocrine Tumor Society; 2012. Available: www.nanets.net/nanets_cd/2012/pdfs /C33singhresources-abstract3.pdf 2012 (accessed 2013 Dec. 3).

4. Singh S, Saskin R, Lin N, et al. 2012 Neuroendocrine Tumor Symposium abstracts: epidemiology of neuroendocrine tumors in Ontario: a 15-year population-based study. Vancouver (WA): North American Neuroendocrine Tumor Society; 2012. Available: www.nanets.net/nanets cd/2012/pdfs /C32singhepi-abstract1.pdf (accessed 2013 Dec. 3).

\section{CMAJ 2014. DOI:10.1503/cmaj.114-0022}

Sickle cell disease: In response to the CMAJ editorial on neglected diseases, ${ }^{1}$ I would like to draw attention to sickle cell disease.

Now is an exciting time to be involved in both the research of sickle cell disease and the care of individuals with the disease. With changes in patterns of immigration, this condition is becoming more prevalent across all regions of Canada. In recent years, a dramatic increase in the understanding of the associated pathophysiology and genetics has led to excellent childhood interventions that have a substantial impact on morbidity and mortality. With the use of penicillin prophylaxis and childhood immunization, sickle cell disease has become a lifelong disorder rather than an indicator of early childhood death. Blood transfusions have effectively combatted the high prevalence of stroke in children with sickle cell disease, thereby reducing disabilities.
These successes bring new challenges. All medical practitioners in Canada - regardless of their geographic location and working environment (e.g., emergency department, family medical office, hospital) must be aware of sickle cell disease and be willing and competent to participate in the management of affected individuals. This single gene blood disorder is now considered a phenotypically variable, multisystem disease caused by chronic ischemia-reperfusion injury and vascular endothelial remodelling. As such, it is an ideal disease to facilitate research across various medical specialties and a breadth of basic science realms in an era of collaboration and discovery. Its genetic basis also permits next generation genomics to further enable future progress and understanding.

The Canadian Haemoglobinopathy Association is composed of a multidisciplinary group of health care providers dedicated to the advancement of sickle cell disease in Canada. We will issue the first Canadian standards for the care of affected patients in Canada. This is an opportunity to educate the wider health care community about this chronic multisystem blood disorder. I encourage $C M A J$ to join us in this work.

\section{Richard Ward MD}

Hematologist, University Health Network and University of Toronto, Toronto, Ont.

\section{Reference}

1. Kelsall D. With a disease for every day, who will care for the orphans? CMAJ 2013;185:1475.

CMAJ 2014. DOI:10.1503/cmaj.114-0023

Cardiac tumours: I would like to commend Kelsall's ${ }^{1}$ initiative to disseminate knowledge on rare diseases. The initiative will certainly help identify, stimulate, coordinate and support research, which could benefit patients who have any one of the nearly 6800 known rare diseases.

I would like to draw attention to tumours of the heart. Primary cardiac 